\title{
LAICIDAD COMO PIEZA CLAVE PARA EL AVANCE DEL ALTERMUNDISMO DESDE LA PERSPECTIVA DE GÉNERO
}

\author{
SECULARISM AS A KEY PIECE \\ IN THE ALTERGLOBALIZATION ADVANCING FROM \\ THE GENDER PERSPECTIVE
}

\author{
Lorena GARCÍA \\ Instituto La Talaia, Tarragona
}

Recibido: $1 / 6 / 2016$

Aceptado: 14/11/2016

Para citar este artículo / To cite this article:

García, Lorena. «Laicidad como pieza clave para el avance del altermundismo desde la perspectiva de género». En Nieves Montesinos Sánchez y Beatriz Souto Galván (coords.), Laicidad y creencias. Feminismo/s, 28 (diciembre 2016): 75-90, DOI: $10.14198 / \mathrm{fem} .2016 .28 .03$

Para enlazar con este artículo / To link to this article:

http://dx.doi.org/10.14198/fem.2016.28.03

\section{Resumen}

El Foro Social Mundial (FSM) es un espacio de encuentro donde los movimientos sociales buscan crear un sistema alternativo a la globalización capitalista. Está amparado bajo la Carta de Principios que, entre otros aspectos, destaca la no confesionalidad como elemento a tener en cuenta a la hora de buscar alternativas al neoliberalismo que domina el mundo. Al mismo tiempo, se da la paradoja que esta cita mundial alberga actitudes patriarcales en su seno.

Este estudio analiza desde una perspectiva crítica de género las diversas ediciones del Foro y, al mismo tiempo, resalta los logros que las mujeres -gracias a las acciones impulsadas por los Feminismos poscoloniales y el trabajo 
previo de los Diálogos Feministas (DF)- han obtenido a lo largo de esta cita mundial mediante el impulso de un ambiente laico, necesario para que las cuestiones de género no sean elemento transversal, sino "capilar», aspecto clave para crear un mundo más justo e igualitario, otro mundo posible.

Palabras clave: Laicidad, Foro Social Mundial, Feminismos poscoloniales, Capilaridad, Igualdad.

\begin{abstract}
The World Social Forum (WSF) is a meeting place where social movements seeking to create an alternative to the capitalist globalization system, protected under the Charter of principles which, inter alia, stresses the need for the non-confessionalism as element to keep in mind when looking for alternatives to the neoliberal system that dominates the world. At the same time, the paradox which houses patriarchal attitudes in their midst is given.

This study analyzes a critical gender perspective the various editions of the Forum, and at the same time, highlights the achievements that women - thanks to the actions promoted by the postcolonial Feminisms and the work of the feminist dialogues (DF) - have gained throughout this event world through the promotion of a secular environment, so that gender issues are not cross element, but «hair», key aspect to create a more just and egalitarian world another possible world.
\end{abstract}

Keywords: Equality, World Social Forum, Post-colonial Feminisms, Capillarity, Secularism. 


\section{FORO SOCIAL MUNDIAL (FSM) Y PRESENCIA DE LOS FEMINISMOS. PAPEL DE LO LAICO}

\subsection{Articulación del Foro Social Mundial (FSM), los Feminismos y la laicidad}

La laicidad debe ser un elemento más que favorezca la igualdad, aspecto que los Feminismos promulgan con el objetivo de que el género sea el elemento central sobre el que pivote el cambio para transformar el actual sistema globalizado y neocapitalista cuya violencia golpea, principalmente, a las mujeres. Estas reivindicaciones están recogidas en el seno del Foro Social Mundial (FSM), que aparece en los albores del siglo XXI con el objetivo de elaborar estrategias de resistencia frente al modelo dominante y de potenciar unas políticas altermundistas que promuevan la equidad y la justicia social.

Este artículo analiza la relación y el peso que tiene la laicidad y el género dentro del FSM y muestra cómo, pese a ser un camino lleno de luces y sombras, es al mismo tiempo un sendero en el que avanza la labor de ambos conceptos y se mantiene la esencia de esta cita mundial.

El Foro Social Mundial nació en 2001 y se define como un espacio democrático donde debatir ideas, analizar, formular proposiciones, intercambiar experiencias y articular movimientos sociales, redes, ONGs y otras organizaciones opuestas al neoliberalismo, capitalismo e imperialismo. Los tres primeros Foros, realizados en Porto Alegre (Brasil), tuvieron gran acogida. Posteriormente se ha celebrado también en otras zonas del mundo. Por otra parte, el Foro tiene sus versiones locales o temáticas en diversas partes del mundo, que se celebran periódicamente.

$\mathrm{Al}$ respecto, los Feminismos apuestan desde el principio por estar presentes dentro del Foro, ya que consideran que es un espacio de aprendizaje para construir movimiento y crear alianzas.

De todos modos, cabe señalar que, paradójicamente, pese a la promulgación que hace el FSM de «otro mundo posible» como lema principal, alberga actitudes patriarcales, aunque al mismo tiempo permite el desarrollo de mecanismos para el debate, la reflexión y el análisis que promuevan el cambio a nivel interno y externo, tratando de que el género vaya teniendo mayor presencia. El 
objetivo por parte de los movimientos feministas es que éste sea una cuestión vertebradora para el cambio.

Por otra parte, hay que señalar que las feministas han sacado a la palestra las reflexiones y las acciones políticas en materia de sexualidad y derechos reproductivos, lo que ha llevado a la creación de un nuevo campo de derechos que debía experimentarse como parte de la ciudadanía. Para el feminismo, el control sobre el cuerpo y lo sexual ha sido desde el comienzo un campo de análisis y de lucha política, que sólo puede ser tratado alejado de las influencias religiosas, tal como se postula en la Carta de Principios del FSM. Ésta es el paraguas en el que se amparan las diversas posiciones que confluyen en el Foro y común denominador de la «búsqueda y construcción de alternativas a la globalización neoliberal del capitalismo» (Calvo 37). Concretamente, en su artículo 8 , se posiciona respecto a lo religioso y resalta que:

El Foro Social Mundial es un espacio plural y diversificado, no confesional, no gubernamental y no partidario, que articula de manera descentralizada y en red a entidades y movimientos que estén involucrados en acciones concretas por la construcción de un mundo diferente, local o internacional.

Y es que el laicismo es sinónimo de defensa del pluralismo, de autonomía del orden jurídico y político, de la dignidad y legitimidad de una moral autónoma y de la libertad de conciencia, como señala Pagola (2011), que añade que también es fuente para reivindicar una cultura de tolerancia activa. Por tanto, la defensa de la laicidad para el desarrollo de las luchas altermundistas es clave no sólo para los Feminismos sino para los movimientos sociales que se amparan en el seno del Foro.

En este sentido, Elsa Conde subraya en el Foro sobre la consolidación de la Laicidad en el Estado mexicano- realizado al amparo del Foro Social Mundial (2007)-, que dicho concepto de laicidad se entiende bajo los principios de respeto a la libertad de conciencia y a su práctica individual y colectiva; la autonomía de lo político y de la sociedad civil frente a las normas religiosas y filosóficas particulares, así como la igualdad ante la ley y la no discriminación directa e indirecta hacia las personas. (Torres 2008).

Desde los inicios del FSM, se aprecia como el movimiento feminista trata de reivindicarse y reivindicar sus derechos en su seno desde la óptica de lo laico. Concretamente, en la II edición del Foro -realizado en Porto Alegre-, la Articulación Feminista Marcosur (AFM) lanzó una campaña bajo el lema «Contra los fundamentalismos lo fundamental es la gente» ${ }^{1}$. Según destacaba Garrido, miembro de la citada organización, su objetivo era ampliar las voces

1. Ver http://www.mujeresdelsur-afm.org.uy/documento-de-la-campana. 
que se oponían a prácticas, discursos y representaciones sociales discriminatorias que sometían a las personas a situaciones de opresión, por lo que además de ir contra el fundamentalismo religioso iba contra el autoritarismo y el patriarcado (61).

Dicha campaña señalaba que todos los fundamentalismos convergían en tratar de imponer su verdad única y una única voz para dominar, controlar y sujetar violentamente los cuerpos, las sexualidades, las subjetividades y las vidas de las mujeres, con la idea de destituirlas de sus derechos humanos, de su derecho al placer, de ejercer libremente su sexualidad, a decidir abortar o a ocupar un espacio de poder.

Esta iniciativa también resaltaba que los proyectos y prácticas que garantizan supremacías se asientan bajo las bases del androcentrismo y el etnocentrismo -blanco, masculino occidental y heterosexual-y sobre la incapacidad para el diálogo, la negociación y la inclusión.

Por tanto, según destaca Garrido, ante los fundamentalismos es necesario construir nuevos paradigmas simbólicos y políticos con los que afirmar las formas democráticas y pacíficas de enfrentar los conflictos, que habiliten el reconocimiento de las diferencias y el ejercicio de la solidaridad en la búsqueda de la convivencia humana, porque el radicalismo, sea religioso, político, científico o cultural, es «siempre político y supone una negación de la pluralidad y diversidad a la vez que legitima mecanismos violentos de presión de un grupo por parte del otro» (61).

Por ello, los Feminismos optan por denunciar el sufrimiento y la violencia ejercida sobre el cuerpo de las mujeres, las formas de represión sexual y la división sexual del trabajo como elemento estructurador de las relaciones de género, con el objetivo de promover una transformación social, al tiempo que «colocan también el debate sobre la esfera política como campo laico. Las feministas lo hacen en el interior del propio Foro» (Betania 14), creando tensiones con otros movimientos y partidos políticos, fruto de la influencia de las iglesias sobre la esfera de la política.

De este modo, los Feminismos exponen en el FSM sus reivindicaciones, comparten sus experiencias con otros movimientos en un espacio y una acción más global pero, pese a los avances, sigue habiendo una jerarquización de las luchas que siempre deja en segunda posición las cuestiones de género ante otras consideradas más urgentes y prioritarias, como el imperialismo, la desocupación y la pobreza.

La palabra «género» está presente en los documentos y programaciones de los diversos Foros y las mujeres participan por medio de propuestas marcadas por la horizontalidad y el diálogo, pero no es el hilo conductor que articule 
las principales preocupaciones. Wilson destaca que los líderes del Foro han sido predominantemente hombres y entre las políticas centrales del Foro, los Feminismos sólo están presentes a nivel teórico en textos, publicaciones y conferencias (14-16).

Pese a todas las dificultades señaladas, los movimientos feministas consideran que el FSM ha estimulado la práctica de una democracia real y participativa por medio de la autogestión, la diversidad, la pluralidad y el consenso, y ahí es donde los Feminismos -especialmente los poscoloniales- han aportado desde sus experiencias muchas de las ideas que el Foro defiende, buscando una nueva manera de ver el mundo, dejando atrás visiones neocapitalistas que mantienen mecanismos de poder y opresión, silencio y exclusión.

Por eso, los Feminismos ven necesario alzar su voz desde su experiencia local sumada a la experiencia global del FSM ya que es «un espacio donde se está alimentando y potenciando las perspectivas de una nueva hegemonía que traen los movimientos sociales» (Vargas 2010).

\subsection{Influencia de los Feminismos poscoloniales y los Diálogos Feministas (DF) en el FSM desde la perspectiva de género y de lo laico}

Los Feminismos poscoloniales son claves, por un lado, para tratar que las reivindicaciones desde la óptica de género tengan mayor protagonismo en el FSM y, por otra parte, para rechazar el androcentrismo y etnocentrismo al que se hacía referencia en la campaña sobre los totalitarismos -entre ellos el religiosollevada a cabo en el II FSM por la Articulación Feminista Marcosur (AFM).

El reconocimiento de los Feminismos poscoloniales rompe con la mirada que el Norte impone como única y unificadora y valora las aportaciones de los movimientos del Sur y de las diferencias en la transformación del modelo global dominante, respetando su voz. Llevado al espacio del Foro, "permitió extender e intensificar debates antes muy limitados como la mercantilización del cuerpo y la vida de las mujeres» (Faria 6).

Los Feminismos poscoloniales tienen como antecedente la labor de empoderamiento que los Feminismos de América Latina y el Caribe realizaron en los años 80 y 90 ante la creciente dominación del discurso capitalista y globalizador, que reducía a las mujeres de estas zonas a meras receptoras de ayudas de gobiernos, instituciones y ONGs como garantes de sus derechos,- lo que se tradujo en un feminismo institucionalizado-que las invisibilizó.

Esta concienciación provocó que movimientos como la Red de Mujeres Transformando la Economía (REMTE), la Articulación Feminista Marcosur (AFM) o la Marcha Mundial de las Mujeres (MMM) se implicaran en el Foro y, al mismo tiempo, participaran junto con otras entidades en los «Diálogos 
Feministas» (DF). Éstos son un espacio de reunión donde las redes internacionales de mujeres debaten, intercambian experiencias y fomentan nuevas relaciones con otros movimientos sociales. Además, Conway resalta como los DF contribuyen a construir otro tipo de internacionalismo feminista a través de su atención concreta a cuestiones de interés para las mujeres pobres y marginadas (43).

Asimismo, cabe recordar que Mohanty sentó las bases de los Feminismos poscoloniales con su artículo Bajo los ojos de Occidente: Academia feminista y discurso colonial (1984). Identificaba mecanismos perpetuadores de estrategias coloniales que calaban en las categorías analíticas de mujer y las representaba como víctimas del sistema patriarcal. La autora exigía considerarlas como sujetos de acción política, constreñidos por estructuras de dominación como el racismo, la pobreza o el género. Posteriormente, revisó este artículo y en su libro Feminism without borders (2003) llamó a construir alianzas híbridas, multiclasistas y transnacionales políticas, haciendo de la diversidad un arma de lucha para construir un feminismo antiimperialista y neoliberal. Apostaba por articular lo universal y lo particular para potenciar un movimiento feminista trasformador y subrayaba que los discursos globalizadores estaban masculinizados, al mismo tiempo que alertaba de la necesidad de vigilar los de los movimientos antiglobalización.

si bien he propuesto que las feministas necesitan ser anticapitalistas, ahora propongo que los activistas y teóricos de la antiglobalización también necesitan ser feministas. El género queda ignorado como categoría de análisis y como base organizativa en la mayor parte de los movimientos en contra de la globalización, y la antiglobalización (junto con la crítica anticapitalista) no parece ser una pieza central en los proyectos feministas de organización, sobre todo en el Primer Mundo / Norte. (Mohanty 450-451)

Por eso, defendía «la centralidad del género» como elemento clave en los procesos de reestructuración globales y clasificó los movimientos sociales como sitios decisivos para construir conocimientos, comunidades e identidades. Pero ya se ha visto que la política del FSM está abierta al Feminismo, pero no marcada.

Pese a eso, Franzway subraya que «es vital que el género sea parte integrante de las campañas políticas por la justicia global. A pesar de sus limitaciones, algunas feministas vieron que el Foro Social Mundial podría ser un sitio importante para el activismo feminista» (9), ya que apoyaba los objetivos de lucha contra la globalización, lo que motivó a la puesta en marcha de los citados Diálogos Feministas. 
Los DF comenzaron previos al inicio del IV FSM, que tuvo lugar en Mumbai (India) y, posteriormente, se fueron sucediendo en otras citas de este evento mundial. En esta línea, González subraya sobre dichos encuentros que son espacios globales y en permanente construcción, de gran potencialidad para revitalizar el movimiento feminista trasnacional e internacionalista, y conectar experiencias de las mujeres, fomentando nuevas relaciones entre las feministas y otros movimientos sociales (14). Esto supone desplazar la mirada hegemónica del Norte sobre las formas de construir Feminismos.

Desde el inicio de los DF se realizaron discusiones alrededor de algunos ejes de análisis que, con matices, se han mantenido en los tres Diálogos Feministas realizados -la globalización neoliberal, los fundamentalismos y los militarismos-, reflejando hasta qué punto éstos son trabajados como un proceso en construcción.

En los I Diálogos Feministas - antesala del IV FSM (Mumbai)- se discutió sobre la división local/global, los temas de derechos humanos y derechos de las mujeres, el cuerpo, los derechos sexuales y los derechos reproductivos. En los II DF-realizados antes del V FSM (Porto Alegre)- los temas fueron: desafiando el neoliberalismo, los fundamentalismos religiosos y la militarización. En los III Diálogos Feministas- celebrados previo al VII (Nairobi) - se mantuvieron los ejes citados anteriormente con la novedad de que introdujeron un marco para estas discusiones: la democracia radical.

De este modo, la postura no confesional de los Diálogos Feministas queda patente mediante los ejes trabajados en las citas comentadas anteriormente, yendo parejos a las transformaciones y convulsiones que el Foro sufrió en las citas clave de 2004 y 2007 (Mumbay y Nairobi) sobre la necesidad de lo laico para el desarrollo de las aspiraciones no sólo del FSM si no de los Feminismos.

En este sentido, los DF pretendían ligar los temas de la denominada «agenda de las mujeres» con discusiones más estructurales sobre el modelo de democracia, el impacto de la globalización neoliberal, el militarismo y los fundamentalismos. De hecho, son «un espacio que quiere demostrar que la inclusión de los llamados temas de las mujeres, -pese a que la búsqueda del consenso, a veces hace perder claridad y consistencia en su contenido-, es inexorablemente parte de una agenda democrática radical» (González 15).

Así que Ruíz de Giovanni considera que «el desafío es mantener una agenda que refleje y haga converger las diversas expresiones de los movimientos. El desafío es radicalizar el método», ampliando también «la participación para incorporar a experiencias de lucha que no se representan a partir de los criterios tradicionales» y oponiéndose a «circunscribir la cuestión de género a momentos y espacios específicos» (44-46). 
Por tanto, la óptica de género que aportan los Feminismos poscoloniales y los Diálogos Feministas dentro del FSM en su relación y diálogo con otros movimientos sociales hace que se reflexione sobre la relación entre producción, reproducción y sexualidad como elementos que «forman parte de los planes simbólico y materiales de las relaciones sociales de explotación y dominación» (Betania 14), algo que sólo se puede abordar desde la laicidad, tal y como se señala por parte del Foro Social Mundial y del movimiento feminista, que aspira a que esta cuestión sea pieza central en el motor del cambio tanto en el seno del ámbito altermundista como del mundo globalizado neocapitalista y liberal.

\section{CITAS CLAVE DEL FSM DESDE LA PERSPECTIVA DE LO LAICO: MUMBAY Y NAIROBI}

Dentro de esta reflexión entre la relación del Foro Social Mundial y los Feminismos y el papel que tiene el laicismo para trabajar en los objetivos previstos por ambos actores, hay que tener en cuenta dos ediciones de esta cita que fueron claves: el IV FSM en Mumbay (2004) y el VII FSM de Nairobi (2007).

Previos a la celebración de ambos Foros tuvieron lugar los I y III Diálogos Feministas, respectivamente, que sirven como referente para ver cómo y cuánto caló el discurso de los Feminismos poscoloniales en estas citas mundiales, marcadas desde lo laico por diferentes motivos.

\subsection{FSM (Mumbay). Apertura a nuevas realidades y fortalecimiento de la presencia de los Feminismos}

En el caso del IV FSM (Mumbay, India), se incluyeron nuevos temas y realidades y supuso la internacionalización y desoccidentalización del Foro. Centenares de activistas, la mayoría indios y de los sectores excluidos (mujeres, dalits ${ }^{2}$, campesinos/as, ...), mostraron su rechazo a la globalización en las calles. Por ello, «las propias características y problemáticas indias obligaron a la inclusión de nuevos temas como la cuestión de la exclusión y la dignidad» (Vivas 12), fortaleciendo vínculos entre lo local y lo global.

Entre los temas tratados con una mayor relevancia en esta cita, cabe destacar el de la privatización del agua, la seguridad alimentaria, las cuestiones de género, el desmantelamiento del Estado, la guerra o la Organización Mundial del Comercio. También se celebró el Foro de la Dignidad Mundial, que hizo

2. En el sistema de castas de la India es lo que se conoce como un paria o intocable, ocupa el escalafón más bajo en la escala social hindú. 
un llamamiento a la lucha contra el sistema de castas que tantas desigualdades y discriminaciones provocaba.

Además, en este IV FSM las reivindicaciones de género junto con la denuncia de los fundamentalismos -entre ellos el religioso-, comenzaron a adquirir más peso gracias a la labor previa a esta cita mundial realizada en los Diálogos Feministas (DF), de los que se ha hablado en el anterior apartado.

Concretamente, este I DF elaboró la declaración «Construyendo Solidaridades», en la que explicaban los motivos de su creación, su papel en el FSM y su posicionamiento ante las cuestiones de género como elemento clave para el cambio.

Entre otros aspectos, mostraba su disposición a desafiar «las actitudes políticas nacionalistas que reivindican cierta casta, raza, etnia, identidad cultural, sexual o religiosa como superior, creando definiciones excluyentes de ciudadanía, señalando enemigos dentro y fuera del Estado, y socavando la solidaridad $»^{3}$.

Recuerda que el patriarcado está presente en la esfera pública y privada, y considera «que los fundamentalismos religiosos, exacerbados por la globalización neoliberal, como un peligroso ataque contra los derechos de las mujeres y contra la democracia secular» ${ }^{4}$.

Todo este caldo de cultivo supuso un salto exponencial respecto a las tres anteriores ediciones del FSM- todas ellas realizadas en Porto Alegre-, sobre los que Vivas subrayaba que «a pesar de la numerosa presencia de mujeres y jóvenes, éstas contaron con muy poca visibilidad y peso político» (11).

De hecho, la Asamblea de Movimientos Sociales - un encuentro dentro del FSM al que acuden libremente las entidades participantes que lo considerenrealizó un llamamiento ${ }^{5}$ al final de este Foro y, en el documento que elaboró a tal efecto, se identificaba con las luchas de los movimientos y organizaciones populares de la India. Al tiempo, condenaba a las fuerzas políticas e ideologías que promovían la violencia, la exclusión, el sectarismo y el nacionalismo, basado en la religión y en la etnicidad.

Además, denunciaba la discriminación por castas, clases sociales, religión, género, orientación sexual e identidad de género; condenaba la perpetuación de la violencia y a opresión contra las mujeres a través de patrones y prácticas culturales, religiosas y tradiciones discriminatorias y llamaba a una jornada de movilización por la inclusión social.

3. Ver http://www.mujeresdelsur-afm.org.uy/dialogos-feministas/92-declaracion-contruyendo-solidaridad-dialogos-feministas-4to-foro-social-mundial.

4. Íbidem.

5. Ver http://www.movimientos.org/es/dss/show_text.php3\%3Fkey\%3D2570. 
Pese al éxito obtenido en cuanto a la presencia y visibilización de las mujeres y su discurso desde la perspectiva de género y de lo laico, Ruíz di Giovanni -miembro de la MMM- señalaba tras el IV FSM que había que dar un paso más, trabajar por un Feminismo «que se articule a partir de las luchas reales de las mujeres y a favor de la autonomía y la igualdad. Esto nos exigió estar presentes en debates donde las feministas no son esperadas, a veces no demasiado bienvenidas» (45), proponiendo visiones y estrategias para la transformación en todos los campos de la lucha por un cambio radical de la sociedad.

Esta autora sostiene que los Feminismos apuestan por estar en todos los rincones del FSM, más en la «capilaridad» que en la «transversalidad», por lo que es necesario que el proceso del Foro no sólo interpele a las autoridades competentes de la globalización, sino que sea capaz, como espacio de convergencia, de incidir también en las prácticas, los códigos y la conciencia de quienes se proponen cambiar de mundo y reconozca que «hay que arriesgarse a construir nuevas referencias capaces de enfrentarse a los paradigmas omnipresentes el imperialismo neoliberal» (46).

Esta reflexión remite nuevamente a la necesidad de establecer el género como elemento «capilar» en el discurso antiglobalización, y entronca con la «centralidad de género» que Mohanty promulga desde la óptica de los Feminismos poscoloniales y de lo no confesional como elementos clave para el desarrollo de las libertades colectivas desde la óptica de género, una labor que empezó a ver sus primeros frutos y visualizarse con las acciones realizadas en este FSM a raíz de la labor previa de los DF.

\subsection{FSM (Nairobi). Contraejemplo del FSM que contribuye a fortalecer el papel de lo laico}

Por otro lado, esta cita mundial se realizó en 2007 en la localidad africana de Nairobi (Kenia). Fue un Foro muy institucionalizado y poco orientado a la acción y supuso «un contraejemplo de cómo debería ser el FSM» (Antentas 30 ), pese a que se siguió apostando por la participación de los movimientos sociales y actividades autogestionadas.

Previo a este FSM tuvieron lugar los III Diálogos feministas, en los que se creó la «Carta de principios feministas para las feministas africanas $»^{6}$. Entre ellos, se recogía la necesidad de abogar por la libertad de elección y autonomía con respecto a los temas de integridad física, incluyendo derechos reproductivos, aborto, identidad sexual y orientación sexual y de establecer

6. Ver http://www.mujeresdelsur-afm.org.uy/dialogos-feministas/83-3er-dialogo-feministanairobi-2007. 
un compromiso crítico con los discursos de la religión, cultura, tradición y vida doméstica, con la atención enfocada en la centralidad de los derechos de las mujeres, entre otros aspectos.

El objetivo de este Foro era arraigar el proceso en África y servir como un mecanismo de coordinación continental de las resistencias al neoliberalismo en el continente más devastado y pobre del planeta. Esta cita supuso un nuevo punto de inflexión en el desarrollo y evolución del FSM, ya que en él coincidieron diversas circunstancias que hicieron que sus miembros se replanteasen la finalidad del mismo, su organización y metodología, ente otros aspectos, y que a continuación iré detallando.

Concretamente, a nivel político el discurso que reinó durante su desarrollo fue más bien «moderado», debido a la gran visibilidad que tuvieron las actividades y discurso de algunas grandes ONGs, alguna de ellas muy ligadas a sectores eclesiásticos conservadores. Más bien este FSM fue un espacio de «encuentro de ONGs», mientras que el lugar para la confluencia de luchas y organizaciones populares fue muy reducida, de modo que fue un Foro muy institucionalizado y poco orientado a la acción (Antentas 28).

A nivel organizativo tuvo varios aspectos que entran en contradicción con el propio enfoque que el FSM quiere dar en sus encuentros. Los preparativos se dejaron en manos de un escaso número de personas que pertenecían a determinadas ONGs, -pero sin la implicación real de los movimientos populares el país- y hubo una fuerte mercantilización del evento que lo convirtió en exclusivo y elitista, por lo que podía acceder muy poca población local.

En esta edición, también hubo un gran despliegue de iglesias y, dentro de ellas, grupos de iglesias reaccionarias y fundamentalistas para con los derechos de las mujeres y las luchas de orientación sexual. En las reuniones previas a esta cita se había informado de ello pero no se tuvo en cuenta. Como ejemplos, por primera vez se realizó en el FSM una marcha contra el aborto y, en la ceremonia de clausura, cuando habló una lesbiana hubo varios momentos de tensión (Vargas 2007).

Esta situación llevó los movimientos sociales participantes en el FSM a reflexionar sobre el papel de la «evangelización» en esta cita mundial, ya que la presencia de grupos eclesiásticos antidemocráticos va en contra de la metodología misma del Foro. Al respecto, Vargas recalca que las iglesias progresistas en África tienen un papel importante, ya que «en países con débiles sociedad civiles, las iglesias son un catalizador de organización de iniciativas populares» (2007), asumiendo un compromiso social y político contra la pobreza y de respeto a los diferentes posicionamientos y reconociendo como referente la ya citada Carta de Principios. Por ello, matiza que 
«una cosa es la presencia de personas ligadas a las iglesias que aportan a la transformación social, respetando la carta de Principios, y otra es la presencia de aquellas expresiones religiosas y eclesiásticas que tienen una limitada perspectiva de los derechos humanos» (2007).

Pese a todo, la presencia de los movimientos de diversidad sexual en esta edición en África fue significativa. Además, Mbatia e Indusa destacaban una gran participación y presencia visible de grupos de mujeres y feministas, lo que calificaban de «un hecho completamente diferente de lo habitual en las reuniones ordinarias de la sociedad civil de Nairobi» (46), fruto del trabajo de las reuniones previas al Foro con los DF, entre otros aspectos.

Ante las obstáculos encontrados en el desarrollo de este Foro, varios movimientos y entidades hicieron llegar al Comité Internacional (CI) del FSM -que fue creado con carácter permanente y es quien marca las líneas estratégicas del mismo- una carta en defensa de la promoción y potenciación de la lucha por los derechos sexuales y reproductivos, como parte del espíritu democrático del FSM. En ella se destacaba que «las luchas por la construcción de otro mundo solamente pueden ser exitosas sí reconocen la diversidad de identidades y sujetos políticos» y agregaba que «el derecho a la lucha por la autonomía y la libertad de nuestr@s herman@s no es negociable» .

Junto con esta iniciativa, la Asamblea de Movimientos Sociales ${ }^{8}$ realizó al final de este FSM un documento en el que mostraba su lucha contra el neocolonialismo, denunciaba la mercantilización, privatización y militarización que se había tratado de hacer en el FSM y, por otra parte, mostraba su preocupación por la presencia de organizaciones que trabajaban contra los derechos de la mujeres, sectores marginados y contra los derechos sexuales y la diversidad, en contradicción con la Carta de los Principios del Foro.

En ese momento y, tras todo lo comentado anteriormente, se decidió que el FSM tuviera lugar cada dos años, ya que esta fórmula estaba mostrando signos de agotamiento que debían llevar a un análisis profundo del mismo, por lo que así se hizo posteriormente en algunas ediciones ${ }^{9}$.

7. Esta carta se firmó primeramente por el Programa Democracia y transformación Global de la Universidad de San Marcos-Lima, la Articulación Feminista Marcosur, el centro Flora Tristán, ABONG y el instituto Paulo Freire. Posteriormente, recibió un conjunto destacado de adhesiones.

8. Ver http://www.hechohistorico.com.ar/Trabajos/Osal/osal/osal21/org/Documentos21.pdf.

9. Posteriormente, el FSM ha retomado recientemente las citas con carácter anual. 


\section{CONCLUSIONES}

Es imprescindible la conservación del carácter laico de los Estados para la protección de las garantías individuales y para el pleno derecho de la ciudadanía de las mujeres. Por ello, el Foro Social Mundial reconoce este aspecto en su Carta de Principios, que es el elemento inspirador del mismo y que ayuda a guiar y reconducir el trabajo, los objetivos y las estrategias a desarrollar de los movimientos que participan en él, como es el caso de los Feminismos.

En este sentido, el papel de los Feminismos poscoloniales en el FSM es clave ya que -a diferencia de la mirada colonial occidental- reflejan la diversidad y las diferencias de necesidades, prioridades, análisis y posicionamientos de género de las entidades participantes, una labor que se trabaja previamente mediante los Diálogos Feministas y que asienta sus reivindicaciones.

A través de estos encuentros previos al Foro, las voces de los Feminismos poscoloniales encuentran un espacio en el que hacerse oír, visibilizarse y empoderarse. Junto con esto, se establecen estrategias para hacer frente a los fundamentalismos, que focalizan su poder, control y violencia en los cuerpos y los derechos de las mujeres. Contribuyen a potenciar la presencia de la perspectiva de género y sus reivindicaciones en esta cita mundial, además de ser defensores y garantes de lo laico para el desarrollo de sus objetivos y los del propio Foro en sí.

El FSM representa una articulación dialéctica entre la justicia global y el movimiento feminista en particular que no es fácil, ya que implica una doble estrategia de compromiso con la negociación colectiva en las luchas de los movimientos sociales y, a la vez, con la transformación de sus puntos de vista en relación con los Feminismos, el género y la diferencia. Lo que está claro es que si los movimientos feministas no hubieran participado en el FSM la cuestión de género y su incorporación no sería palpable progresivamente ni constaría como tema de discusión.

Por ello, los Feminismos, en sintonía con el FSM, coinciden en tratar de hacer frente a todo tipo de fundamentalismo, que cercena los derechos de la ciudadanía, por lo que la apuesta por la laicidad es una pieza clave para el desarrollo de estrategias que rompan y transformen esta hegemonía religiosa de opresión y exclusión.

Claros ejemplos de lo anteriormente citado son el foro de Mumbay, como exponente del aperturismo a nuevas realidades y sensibilidades dentro de la trayectoria del FSM, y el de Nairobi, como cita en la que se sacudieron los cimientos de este evento para volver a resituar su eje nuevamente en la Carta de Principios. 
En ambas citas los Feminismos, pese a las dificultades, aprovecharon este espacio para hacer oír su voz y para expresar su diversidad y sus reivindicaciones frente al control del cuerpo y la sexualidad. Además, tuvieron como referente en todo momento el carácter no confesional con el que se postula esta cita mundial y que sirvió de guía ante la diversidad en el caso del IV FSM en la India, y de freno ante los intentos de control que se trataron de llevar a cabo autoridades religiosas en el VII FSM, celebrado por vez primera en África.

De hecho, según se han ido realizando nuevas ediciones del Foro se han generado alianzas intramovimientos con un posicionamiento político basado en la autonomía dialogante y negociadora cuya única condición es el reconocimiento de la «otredad» como sujeto de derecho, la equidad como un valor democrático a incorporar como referente y el reconocimiento de la diversidad sexual como un valor y un derecho democrático.

Por tanto, sólo desde lo laico se puede trabajar para responder a las reivindicaciones de los Feminismos y para que éste siga tratando de promulgarse como elemento de transformación «capilar», no sólo en el seno del FSM, si no también fuera del mismo en la relación del Foro Social Mundial y otros movimientos sociales con los Estados.

\section{REFERENCIAS BIBIOGRÁFICAS}

Antentas, José M. ${ }^{a}$ «Proceso del FSM en África: una dinámica de arriba abajo». El futuro del Foro Social Mundial. Retos y perspectivas después de Nairobi. Ed. Josep M. ${ }^{a}$ Antentas et al. Icaria, Barcelona: 2008, 55-64.

Betania, Maria. «Una mirada feminista sobre el Foro Social Mundial.» Reflexiones colectivas, escrituras horizontales (2010): 7-18.

Calvo, Jorge. «El Foro Social Mundial: qué es y cómo se hace» (2007). Disponible en: <http://www.comitesromero.org/jornadas/lleida2008/documentos/viernes4juliodocumentos/elfsmqueesycomosehacejordi\%20calvorufanges.pdf>, consultado el 20-05-2016.

Conway, Janet. «Activist knowledges on the anti-globlalization terrain: transnational feminisms at the World Social Forum». A journal for and about social movements Article 3.2 (2011): 33-64.

Faria, Nalu. «Le féminisme latino-américain et caribéen: perspectives face au néolibéralisme. (2008): 1-7, disponible en: <http://www.google.es/ url $? \mathrm{sa}=\mathrm{t} \& \mathrm{rct}=\mathrm{j} \& \mathrm{q}=\& \mathrm{esrc}=\mathrm{s} \&$ source $=\mathrm{web} \& \mathrm{~cd}=1 \& \mathrm{ved}=0 \mathrm{CCAQFjAA \&}$ url=http $\% 3 \mathrm{~A} \% 2 \mathrm{~F} \% 2 \mathrm{Fcadtm}$. org $\% 2 \mathrm{FIMG} \% 2 \mathrm{Fdoc} \% 2 \mathrm{FLe}$ _feminisme_ latinoamericain_et_caribeen__perspectives_face_au_neoliberalisme. doc\&ei=tByiU_TiBqfX0QWW_YDwCQ\&usg=AFQjCNHVFnowDsP1EXoa942SSZqV3LwQ>, consultado el 01-05-2014>, consultado el 1-05-2016. 
Franzway, Suzanne. «Working trought the World Social Forum». (2009): 1-11, disponible en: <http://www.tasa.org.au/conferences/conferencepapers09/papers/ Franzway,\%20Suzanne.pdf>, consultado el 09-05-2016.

Garrido, Lucy. «Los fundamentalistas no se ríen». Porto Alegre (Foro Social Mundial 2002). Una asamblea de la humanidad. Ed. Carlos Albin et al. Icaria, Barcelona: 2002, 60-62.

González, Ana Cristina. «Sobre los Diálogos Feministas». Revista Cotidiano Mujer n. ${ }^{\circ} 44$ (2008): 14-20.

Hewitt, Lindy. «Feminism and the Forum. Is it worth the effort?». Societies Without Borders 3 (2008):118-135.

Mbatia, Wangui e Indusa, Hassan. «El FSM 2007: una perspectiva desde Kenia». El futuro del Foro Social Mundial. Retos y perspectivas después de Nairobi. Ed. Éric Toussain et al. Icaria, Barcelona: 2008, 43-63.

Mohanty, Chandra Talpade. «De vuelta a Bajo los ojos de Occidente: La solidaridad feminista a través de las luchas anticapitalistas». Descolonizando el feminismo: teorías y prácticas desde los márgenes. Eds. Liliana Suárez-Navas y Regina Berger. Madrid: Cátedra, 2008, 404-454.

Pagola, Javier. «El laicismo es emancipación». Alandar, revista de información social y religiosa 277. (2011). Disponible en: <http://www.alandar.org/spip-alandar/?Ellaicismo-es-emancipacion\#.VOYkcISLTIUZ>, consultado el 16-05-2016.

Ruíz de Giovanni, Julia. «Después de Mumbai». Mumbai (Foro Social Mundial 2004). Balance y perspectivas de un movimiento de movimientos. Eds. Esther Vivas et al. Barcelona: Icaria, 2004, 41-49.

Torres, Gladis. «Estado laico, condición para que mujeres ejerzan su ciudadanía». Cimacnoticias (2008). 25 de mayo de 2016. Disponible en: <http://www.cimacnoticias.com.mx/node/48774>, consultado el 25-05-2016.

Vargas, Virginia. «Articulación Feminista Marcosur; Foro Social Mundial 2007». Cotidiano n. ${ }^{o}$ 43.Agenda de las mujeres. (2007). Disponible en: <http:// agendadelasmujeres.com.ar/index2.php?id=3\&nota=4444>, consultado el 14-05-2016.

Vargas, Gina. «Pistas para pensar algunas dimensiones de la nueva hegemonía». Seminario Dez anos. (2010). Disponible en: <http://seminariol0anosdepois. wordpress.com/2010/01/13/pistas-para-pensar-algunas-dimensiones-de-lanueva-hegemonia/s, consultado el 12-05-2016.

Vivas, Esther., et al. Mumbai (Foro Social Mundial 2004). Balance y perspectivas de un movimiento de movimientos. Barcelona: Icaria, 2004.

Wilson, Ara. Feminism in the Space of the World Social Forum. Journal of International Women's Studios núm. 8(3) (2007): 10-27, disponible en: <http:// vc.bridgew.edu/jiws/vol8/iss3/2>, consultado el 15-05-2016. 